\title{
The Role of Solvent Environment on the Optical Behavior of Chemically Synthesized Silicon Nanoparticles
}

\author{
Rawabi M. Al Mohaimeed, ${ }^{1}$ Anees A. Ansari $\mathbb{D}^{2},{ }^{2}$ and Abdullah Aldwayyan ${ }^{1}{ }^{1}$ \\ ${ }^{1}$ Department of Physics, College of Sciences, King Saud University, Riyadh 11451, Saudi Arabia \\ ${ }^{2}$ King Abdullah Institute for Nanotechnology, King Saud University, Riyadh 11451, Saudi Arabia \\ Correspondence should be addressed to Abdullah Aldwayyan; dwayyan@ksu.edu.sa
}

Received 23 November 2017; Revised 8 February 2018; Accepted 20 February 2018; Published 10 April 2018

Academic Editor: Javier Garcia-Guinea

Copyright (c) 2018 Rawabi M. Al Mohaimeed et al. This is an open access article distributed under the Creative Commons Attribution License, which permits unrestricted use, distribution, and reproduction in any medium, provided the original work is properly cited.

\begin{abstract}
Silicon nanoparticles (Si-NPs) were prepared by solution-based chemical etching method. Optical characteristics of the as-prepared Si-NPs were investigated in different polar and nonpolar organic solvents. The emission and absorption properties of Si-NPs were tuned by altering the environment (solvents). The variation in absorption coefficient was observed because of the solvent interaction nature of Si-NPs. Si-NPs in polar aprotic and nonpolar solvents manifested good luminescence under UV excitation. PL intensities were observed to be depending on etched cross-section area on wafer surface. The results show a linear dependence of the refractive index $(n)$ on wavelength $(\lambda)$. The nature of solvents altered the luminescence efficiency of Si-NPs when examining under UV lamp. The emission and absorption properties of Si-NPs were tuned by altering the environment (solvents) through electrostatic interaction of various organic solvents with the Si-NPs. The band shapes of the Si-NPs show remarkable changes in passing from noncoordinating solvent (chloroform) to various coordinating solvents, which was the result of change in the environment around Si-NPs in various solutions.
\end{abstract}

\section{Introduction}

Silicon nanoparticles (Si-NPs) can be classified into a family of discrete sizes that includes $1.0,1.67,2.15,2.9$, and $3.7 \mathrm{~nm}$ diameters [1]. Photoluminescence excitation spectroscopy measured the four smallest highest occupied molecular orbital (HOMO) lowest unoccupied molecular orbital (LUMO) band gaps of 3.44, 2.64, 2.39, and $2.11 \mathrm{eV}$, while emission spectroscopy shows that they are ultrabright UV/blue, green, yellow, and red luminescent particles with band peaks at $\sim 410,540,570$, and $600 \mathrm{~nm}$ $[2,3]$. Furthermore, investigation of Si-NPs in nonlinear optical response under a variety of multiphoton infrared excitation including $1.06,1.3$, and $4.9 \mathrm{~m}$ radiation, requiring 3,3 , and 15 photon processes to achieve direct gap excitation of carriers, the interest has been dampened by the fact that the luminescence is wide band and of low quantum efficiency. Silicon-based nanomaterials are some new photoelectronic and informational materials developed rapidly in recent years. For a long time, silicon has been considered as unsuitable for optoelectronic applications because bulk silicon emits hardly any useful light due to its indirect band gap nature. This opinion was deeply changed after the discovery of bright emission from porous silicon and NPs. The possibility of constructing optoelectronic devices, Si-based integrated circuits, and sensors based on silicon has generated tremendous interest in the preparation and characterization of light-emitting Si-NPs. Although, the first porous silicon layer was observed by Uhlir in 1956 [4] and later by Turner [5], it was only during Canham in 1990 after reporting about room temperature luminescence that there began significant interest in this material [6]. The main obstacle of using SiNPs in optical applications is the much slower recombination rate (excitation lifetime is 4-5 orders of magnitude larger than in some direct band gap quantum dots).

It is fact that the luminescence properties of Si-NPs are size dependent and multicolored, which have exciting potential applications as fluorescent tags for biological imaging [7-9] and a great variety of NPs are currently 
used for bioanalysis including QDs [10]. Si-NPs are much brighter and more stable for photobleaching [11] than organic dyes [12] with broader excitation spectra, which make emission at multiple wavelengths (from particles of different sizes) excited by a single source possible. Si-NPs have a wide range of applications in various fields such as electronics, fuel cells, batteries, agriculture, food industry, and medicines. Si-NPs can vastly improve mechanical, magnetic, electrical, and catalytic properties compared to conventional bulk $\mathrm{Si}$ materials [13-15]. Si-NPs are a promising material to use as biological labels [16]; metal NPs are usually considered harmful as they become transparent to cell dermis. Toxicity of NPs also appears predominant owing to their high surface area and enhanced surface activity. Si-NPs are less toxic than QDs; they offer higher quantum luminescence efficiency than the latter in composites [17], as well as prove to be more stable to the environment.

Previously, some coworkers in our laboratories studied the emission spectra that Si-NPs fabricated by chemical etching technique in different organic solvents $[12,18,19]$. They examine the effects of solvents and temperature on the optical absorption spectra and luminescence spectra of Si-NPs, in which the emission and absorption properties of Si-NPs were tuned by altering the environment (solvents) because of electrostatic interaction of various organic solvents with Si-NPs [19]. The band shapes of the Si-NPs show remarkable changes in passing from noncoordinating solvent (chloroform) to various coordinating solvents, which is the result of change in the environment around Si-NPs in various solutions and suggests coordination of solvent molecule(s), in some cases.

In this study, our main aim is to investigate the optical properties of chemical etching method prepared for Si-NPs in various solvents. We employed various polar and nonpolar organic solvents such as ethanol, DMSO, DMF, dioxane, acetonitrile, chloroform, carbon tetrachloride, benzene, and hexane. Because of their different surface functional groups, they make hydrogen bond with Si-NPs and altered the luminescence efficiency of the nanomaterials. Different parameters including absorption coefficient, reflectance, reflective index, dielectric constant, optical conductivity, and energy band gap were applied to investigate the optical behavior of Si- NPs in various solvents.

\section{Experimental Details}

2.1. Material. Silicon wafers (SUMCO Corporation, Japan), methanol (99.7\%, Sigma-Aldrich, USA), ethanol (95\%, Sigma-Aldrich, USA), acetonitrile (99.8\%, Sigma-Aldrich, USA), chloroform (E-Merck, Germany), carbon tetrachloride (Qualikems, India), isopropanol (Panreac Quimica Co., Spain), benzene (E-Merck, Germany), dioxane (Fisher Scientific Co., UK), hexane (99.9\%, Avonchem, UK), hydrofluoric acid (THF, Panreac Quimica Co., Spain), tetrahydrofuran (THF) (Nexgen Chemicals, India), hydrogen peroxide $\left(\mathrm{H}_{2} \mathrm{O}_{2}\right)$ (Avonchem, UK), dimethyl sulphoxide (DMSO) ( $99 \%$ Fluka Chemika, USA), dimethylformamide (DMF) (99.8\%, Panreac Quimica Co., Spain), chloroplatinic solution, and distilled water (DI) were of analytical grade and used directly as received without any further purification.

2.2. Synthesis of Si-NPs. Silicon NPs were prepared by chemical etching method based according to the published literature reports $[2,20]$. This technique passes through two main steps, namely, the catalyst step and etchant step. In a typical method, Si wafer is cut into pieces typically of about $1 \times 3 \mathrm{~cm}$ in dimension, which were ultrasonically cleaned with acetone prior to drying using nitrogen gas. After that, the wafer was treated in a mixture of $13.5 \mathrm{DI}$, $5 \mathrm{~mL} \mathrm{HF}$, and $1.5 \mathrm{~mL}$ of chloroplatinic solution in polypropylene tank for specified time as follows: $15 \mathrm{~min}, 12 \mathrm{~min}$, $10 \mathrm{~min}, 8 \mathrm{~min}$, and $6 \mathrm{~min}$ to deposit a platinum catalyst. The samples were then rinsed with $\mathrm{H}_{2} \mathrm{O}$ and dried in blowing $\mathrm{N}_{2}$ gas. Wafers were transferred into the etchant tank straight away. Etchant solution was prepared using the $10 \mathrm{~mL}$ methanol, $5 \mathrm{~mL} \mathrm{HF}$, and $10 \mathrm{~mL} \mathrm{H}_{2} \mathrm{O}_{2}$. The wafer will usually bubble if the etching was working. The etched wafers were rinsed then in isopropanol each time and dried slowly with nitrogen gas. To examine if the SiNPs were formed, the etched pieces were tested under UV light. If Si-NPs were present, wafer will glow brightly (in the dark or even in room light).

Spectroline XX-15NF UV lamp was used as the UV light source. It has two wavelengths, one emitting long-wave UV $(365 \mathrm{~nm})$ and the other short-wavelength UV $(254 \mathrm{~nm})$. Then Si wafer is to be immersed into a glass bottle filled with favorable solution such as toluene, benzene, DMSO, DMF, and hexane. The glass bottle was placed in an ultrasound bath for 4 minutes to disperse the Si-NPs. After that, wafers were pulled out and the solution that occurred was loaded with Si-NPs. The differences between the blank solution and Si-NP-dispersed solution were examined by UV light source in a dark room. The obtained NPs were centrifuged and washed for further characterization. This process is considered to be a complete one round of etching. We applied several round to get the Si- NPs until Si wafer turn from mirror surface to black one after etching.

2.3. Characterization. UV/vis absorption spectra were measured using by Perkin-Elmer Lambda- 40 spectrophotometer, with the sample contained in $1 \mathrm{~cm}^{3}$ stoppered quartz cell of $1 \mathrm{~cm}$ path length, in the range $190-600 \mathrm{~nm}$. The emission spectra of Si-NPs were recorded on a PerkinElmer photoluminescence spectrophotometer equipped with a $150-\mathrm{W}$ Xenon lamp as the excitation source.

\section{Results and Discussion}

3.1. Optical Properties of Si-NPs in Various Solvents. Optical absorption spectroscopy was employed to investigate the optical properties of synthesized Si-NPs in the UV-Vis (200-600 nm) region in different solvents after four rounds of wafer etching at room temperature. Figure 1(a) displays the absorption spectra of the as-prepared Si-NPs in different coordinating solvents such as ethanol, dimethyl sulfoxide, dimethyl formamide, dioxane, and acetonitrile. In measuring the absorption spectra of NPs in acetonitrile 


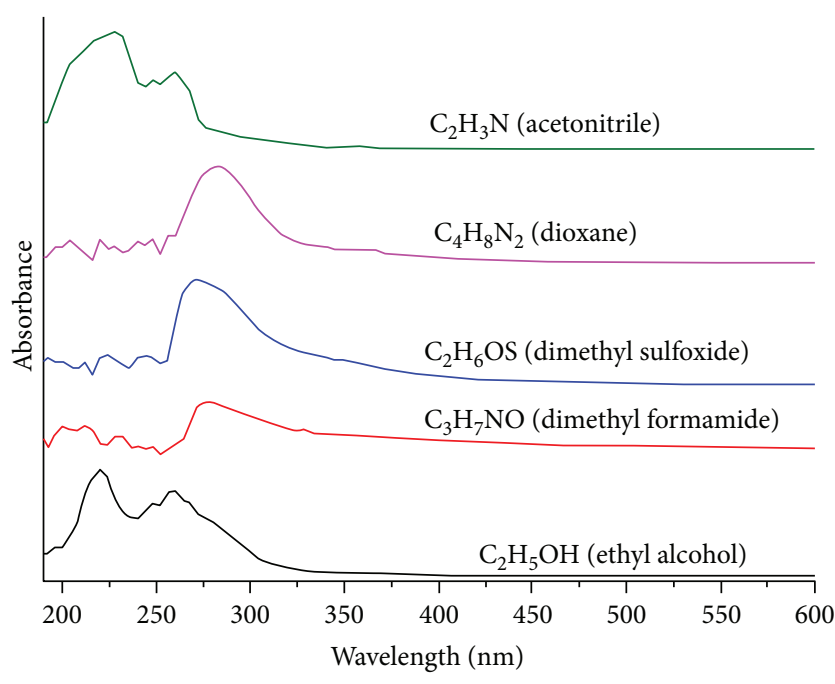

(a)

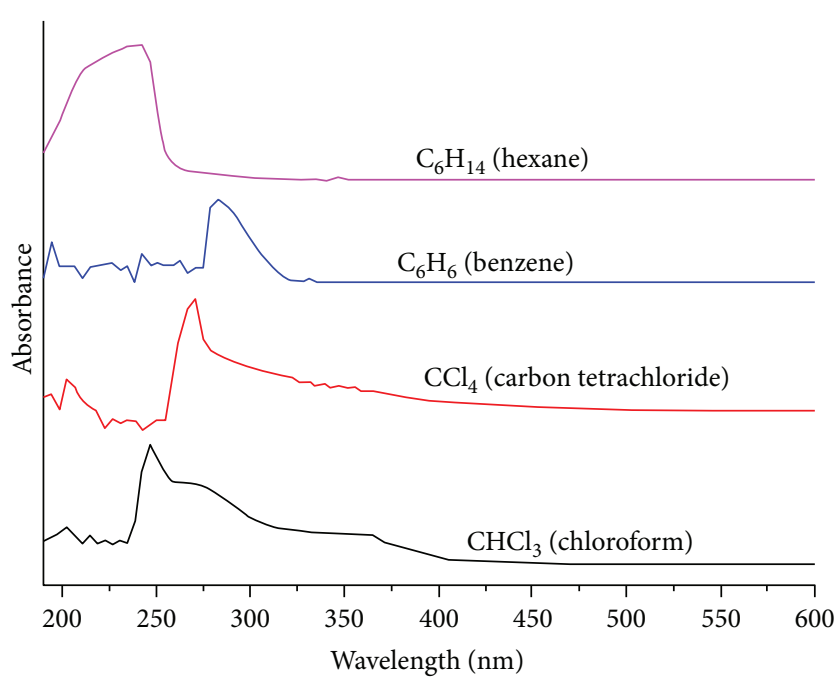

(b)

FIgure 1: Optical absorption spectra of Si-NPs in different solvents.

TABLE 1: List of absorption peaks, emission peaks, and corresponding intensity of the Si-NPs in different solvents.

\begin{tabular}{|c|c|c|c|c|c|}
\hline Solvent & Polarity index & Absorption peak maxima & Absorbance & Emission peaks $(\mathrm{nm})$ & Corresponding intensity \\
\hline Ethanol & 5.2 & $\begin{array}{l}220 \mathrm{~nm} \\
260 \mathrm{~nm}\end{array}$ & $\begin{array}{l}\text { Strong } \\
\text { Shoulder }\end{array}$ & 432.3 & 18,629 \\
\hline DMF & 6.4 & $\begin{array}{l}212 \mathrm{~nm} \\
276 \mathrm{~nm}\end{array}$ & $\begin{array}{l}\text { Weak } \\
\text { Strong }\end{array}$ & 425.2 & 6248 \\
\hline DMSO & 7.2 & $272 \mathrm{~nm}$ & Strong & $\begin{array}{l}430 \\
665\end{array}$ & $\begin{array}{c}63,380 \\
1434\end{array}$ \\
\hline Dioxane & 4.8 & $284 \mathrm{~nm}$ & Strong & 612 & 21,464 \\
\hline Acetonitrile & 5.8 & $\begin{array}{l}228 \mathrm{~nm} \\
260 \mathrm{~nm}\end{array}$ & $\begin{array}{l}\text { Strong } \\
\text { Shoulder }\end{array}$ & 612 & 1508 \\
\hline Chloroform & 4.1 & $\begin{array}{l}248 \mathrm{~nm} \\
260 \mathrm{~nm} \\
332 \mathrm{~nm}\end{array}$ & $\begin{array}{c}\text { Strong } \\
\text { Week } \\
\text { Week }\end{array}$ & $\begin{array}{l}431 \\
617\end{array}$ & $\begin{array}{c}248.20 \\
131.118\end{array}$ \\
\hline Carbon Tetrachloride & 1.6 & $272 \mathrm{~nm}$ & Strong & 603 & 1027 \\
\hline Benzene & 2.7 & $284 \mathrm{~nm}$ & Strong & - & - \\
\hline Hexane & 0 & $244 \mathrm{~nm}$ & Strong & $\begin{array}{l}402 \\
429\end{array}$ & $\begin{array}{l}358 \\
248\end{array}$ \\
\hline
\end{tabular}

and ethanol, this transition is broadened and splitted into two bands with maxima at $228 \mathrm{~nm}$ and $260 \mathrm{~nm}$ and $220 \mathrm{~nm}$ and $260 \mathrm{~nm}$, respectively. Such splitting may be due to the crystal field effect [19]. The absorption spectra in DMSO and DMF show a broad band but in dioxane, this broad band shifted to higher wavelength. The broadening and shifting of the absorption band at lower energy (longer wavelength) are due to coordination of solvent with Si-NPs. Remarkable changes are observed in measuring the absorption spectra in noncoordinating solvents such as chloroform, carbon tetrachloride, benzene, and hexane (Figure 1(b)). It indicates the weak binding of SiNPs with the solvent through hydrogen bonding. In comparing the spectra in different solvents, a remarkable change in band shapes of the transitions is also observed. It specifies the effect of solvent on the optical character of the small-size Si-NPs. It is concluded that the changes in band shapes offer some measure of the relative coordinating ability (complexation) of the solvent to the Si-NPs $[21,22]$. Moreover, the very small difference could be related to different natures of the solvents [21]. The comparative absorption spectral analysis in various solvents is presented in Table 1. It shows that at the same quantity of wafer pieces, the correspondence peak position, band shape, and intensities are influenced based on their respective coordination nature. Variation in shape of absorption bands in different solvents is due to the nature of chemical interaction between Si NPs and solvents. However, absorption intensities are dependent on Si-NP concentration. Additionally, the absorption intensity and concentration of Si-NPs in solution may possibly depend on the crosssectioning of the etched area on silicon wafer. The etched 
surface area may vary in wafer piece to piece because of the nonuniformity etching in HF-based etching solution. The larger etched area of wafers may produce greater quantity of particles in solvents [18].

Photoluminescence spectra were performed to investigate the luminescence properties of the as-prepared SiNPs. The Si-NPs prepared by chemical etching method are optically characterized by suspension in various organic solvents including ethanol, DMSO, DMF, dioxane, benzene, chloroform, $\mathrm{CCl}_{4}$, and hexane. The photoluminescence spectra were recorded over wide range 380$700 \mathrm{~nm}$ wavelengths under monitoring $355 \mathrm{~nm}$ excitation wavelength. In view, recorded emission spectra are recorded after 4 rounds of wafer etching in different solvents using four wafers. The observed photoluminescence spectral results measured in various solvents are summarized in Table 2. The emission spectra of Si-NPs in different solvents show a remarkable difference due to their interaction with solvents. The difference of color in various solvents can be clearly distinguished in Table 2 . It is observed in Table 2 that the wavelength shifts towards a longer wavelength (red shift) when increasing polarity index and dielectric constant of solvents. The intensity of samples can be attributed to the nature of chemical interaction between the Si-NPs and solvent. The emission peak maximum in acetonitrile is observed at $612 \mathrm{~nm}$ (Figure 2(a)). The emission peak position in $\mathrm{CCl}_{4}$ is shifted to a lower wavelength in respect to acetonitrile; it suggests that $\mathrm{CCl}_{4}$ does not interfere in silicon structure whereas acetonitrile interacted with the Si-NPs; therefore, it is shifted at a longer wavelength Figure 2(b). The emission peak positions and peak maxima of the Si-NPs in different solvents are listed in Table 1, where each sample was obtained by etching of 4 wafer pieces and was exposed to $355 \mathrm{~nm}$ excited emission light. It is observed that the emission peak intensity in various solvents is greatly influenced because of the solvent nature. The emission intensities of Si-NPs in solvents possibly depend on the nature of solvents and the cross-section area of etching. Higher cross-section area of etching on wafer surface can produce greater quantity of Si-NPs in solvents.

We expected that the weak emission intensity in solvents could be due to smaller dielectric constant values. Additionally, DMSO reflects high-emission intensity because of large dielectric constant values. These results are consistent with previous published reports [23]. Accordingly, the larger dielectric constant values more than 20, the Onsager length becomes of the order of the nanostructure size, and no further quenching is observed. The Si-NPs in aprotic solvents show good luminescence. The possibility of high luminescent efficiency in unaprotic solvents is because they are not containing the $\mathrm{O}-\mathrm{H}$ functional groups, which help in emitting light of Si-NPs in solvent with stability since aprotic solvents do not contain such functional groups. The Si-NPs exhibit good luminescence spectra in some nonpolar solvents but its stability is very limited [18]. It can be easily discerned from Figure 3 that the absorption band shape and peak maxima of SiNPs in different solvents are increasing with polarity index and dielectric constant. The absorption peak shifts towards a
TABLE 2: Relation between solvent, etching no. of rounds, polarity index, and color of emission for Si NPs under UV excitation.

\begin{tabular}{lccc}
\hline Sample & $\begin{array}{c}\text { Number } \\
\text { of rounds }\end{array}$ & $\begin{array}{c}\text { Polarity } \\
\text { Index }\end{array}$ & $\begin{array}{c}\text { Picture of sample } \\
\text { under UV lamp } \\
\text { (comparing with } \\
\text { pure solvent) }\end{array}$ \\
\hline Ethyl acetate & 4 & 4.4 & \\
\hline
\end{tabular}

\begin{tabular}{lllll}
\hline Benzene & 4 & 2.7 & & \\
Toluene & 4 & 2.4 & & \\
\hline Carbon tetrachloride & 4 & & & \\
\hline
\end{tabular}

longer wavelength (red shift) when increasing polarity and dielectric constant of solvents. As shown in Figures 4(a) and 4(b), the emission peak of Si-NPs in different solvents increases with increasing polarity index and dielectric constant of the solvents and shifted to a longer wavelength (red shift) or a shorter wavelength (blue shift).

3.2. Observed Optical Parameters. The optical absorption coefficient was calculated with the as-prepared samples.

$$
\alpha=\frac{1}{L} \frac{A}{\log e} .
$$

According to the absorption coefficient $(\alpha)$ equation, $\alpha$ is an absorption coefficient, $A$ is for absorbance or optical density of the samples, and $L$ is thickness of the sample. The optical absorption edge was analyzed by (2). In addition, we applied Tauc formula to investigate the electronic band gap structure of the as-prepared Si-NPs, in which band gap energy $\left(E_{g}\right)$ is determined from the energy difference between highest occupied molecular orbital and lowest occupied molecular orbitals. In measuring the $E_{\mathrm{g}}$ values, we utilized the sharply 


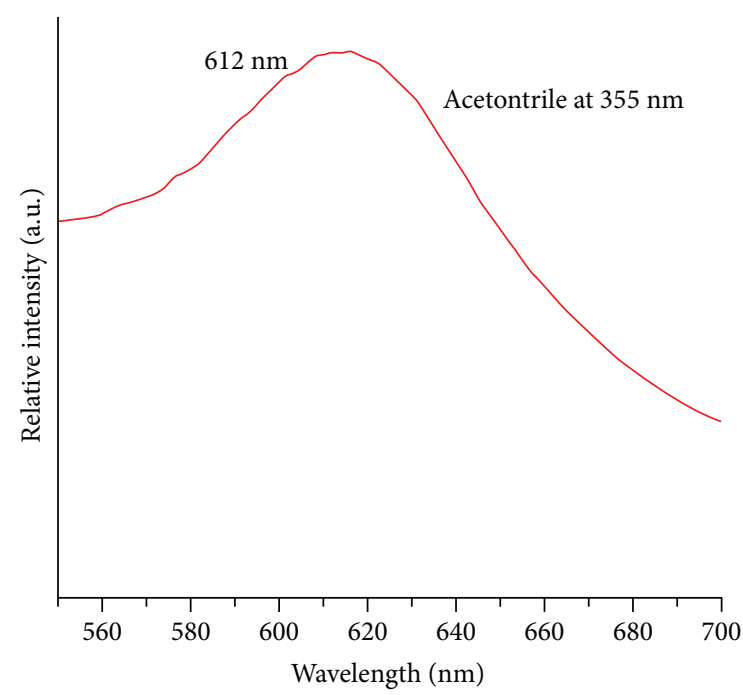

(a)

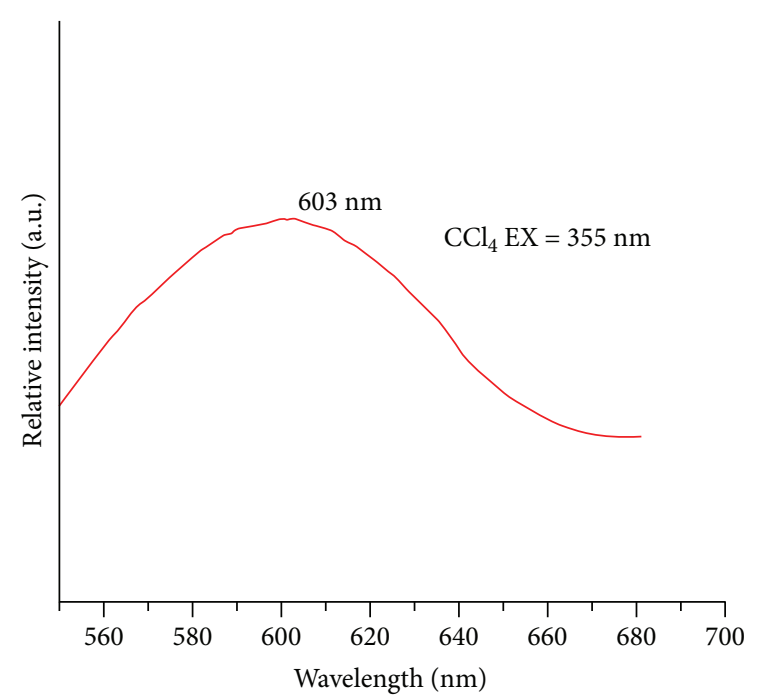

(b)

FIgURE 2: Emission spectra of Si nanoparticle in (a) acetonitrile and (b) carbon tetrachloride.

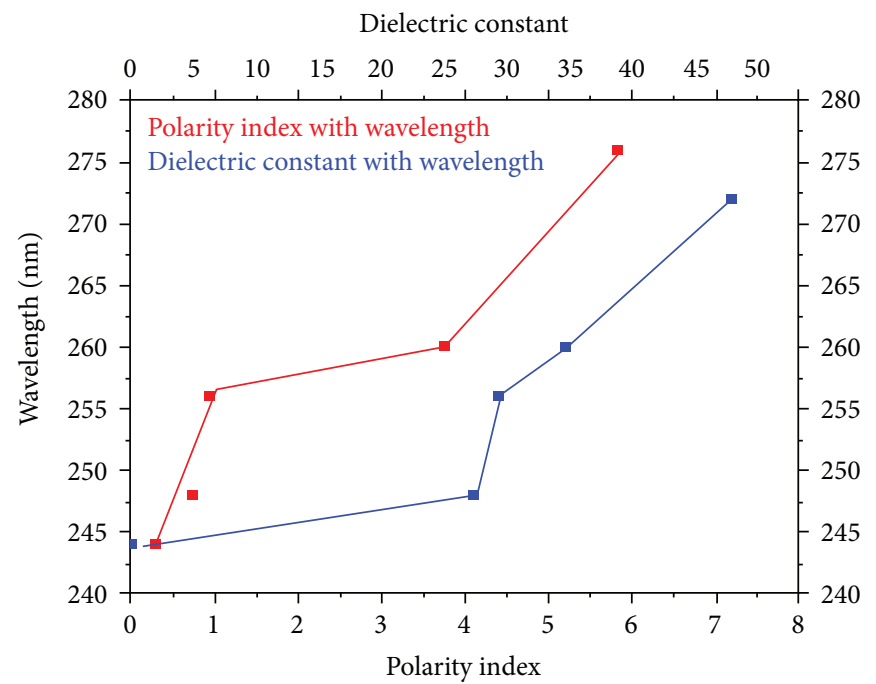

Figure 3: Polarity index and dielectric constant dependence of absorption wavelength of Si-NPs in different solvents.

increasing absorption region of the Si-NPs for determination of optical properties and their correlation between $E_{g}$ and particle size of the samples. The direct band gap was calculated by plotting $(\alpha \mathrm{h} v)^{2}$ as a function of $\mathrm{h} v$ and extrapolating the linear portion of the curve to the $x$-axis. The band gap values $\left(E_{\mathrm{g}}\right)$ of the Si-NPs were determined by optical absorption spectra through Tauc formula [24].

$$
\alpha \mathrm{h} v=\mathrm{A}\left(\mathrm{h} v-E_{g}\right)^{n},
$$

where $\alpha$ is absorbance coefficient and the exponent $n$ depends on the type of transition; $n$ may have values $1 / 2$ and 2 corresponding to the allowed direct and allowed indirect transitions, respectively. Figure 5 shows the $E_{g}$ value for Si-NPs in ethanol and toluene. As observed from the absorption spectra, the absorption edge validated the direct band gap in the as-prepared samples. The $E_{g}$ values are obtained by extrapolating the linear portions of the plots to intercept the photon energy axis, that is, the values of the band gap $E_{g}$ for Si-NPs in different solvents are estimated from the intercept of the obtained straight lines at zero absorption. As shown in Figure 5, the experimentally estimated $E_{g}$ value of Si-NPs in ethanol and toluene is 3.52 and $3.61 \mathrm{eV}$, respectively. We expected that the increasing trend in $E_{g}$ value may be due to the decrease in the particle size.

Reflectance spectra were also measured for other samples by using (UV-VIS spectrophotometers) to find out some optical parameters of Si-NPs in different solvents such as refractive index and dielectric constant. The variation of reflectance $(R)$ as a function of wavelength for Si-NPs in 


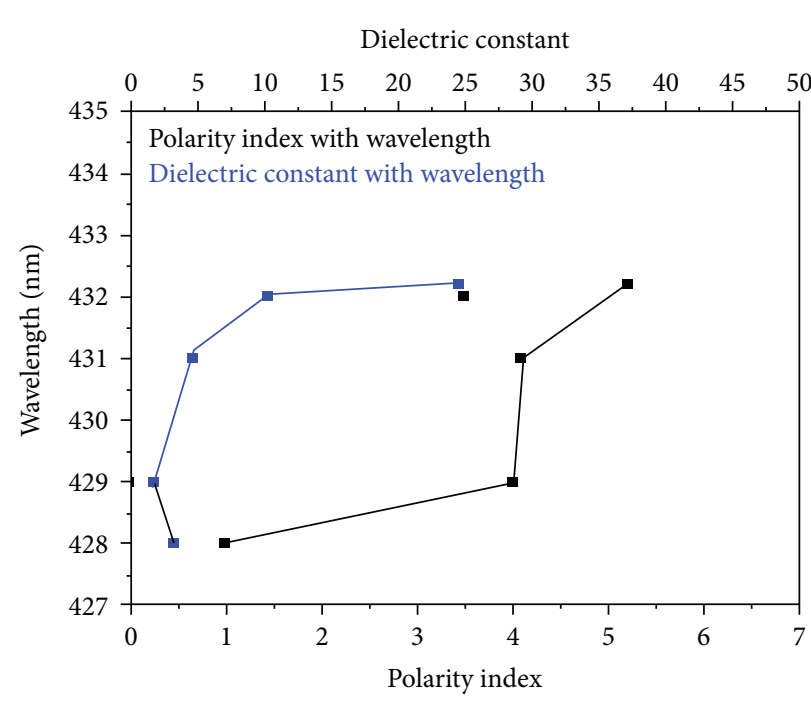

(a)

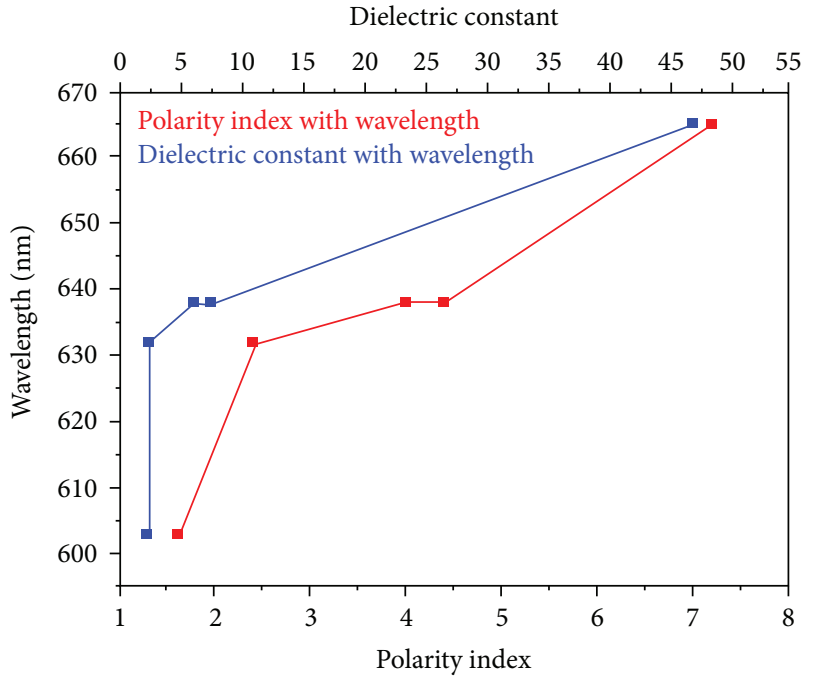

(b)

FIgURE 4: Polarity index and dielectric constant dependence of emission wavelength of Si-NP's (a) blue emission and (b) red emission.

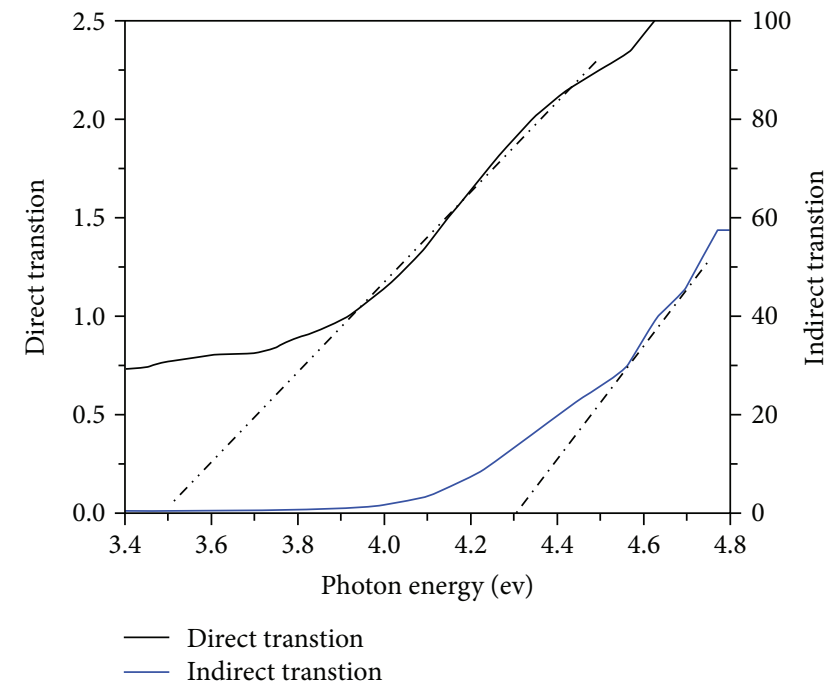

(a)

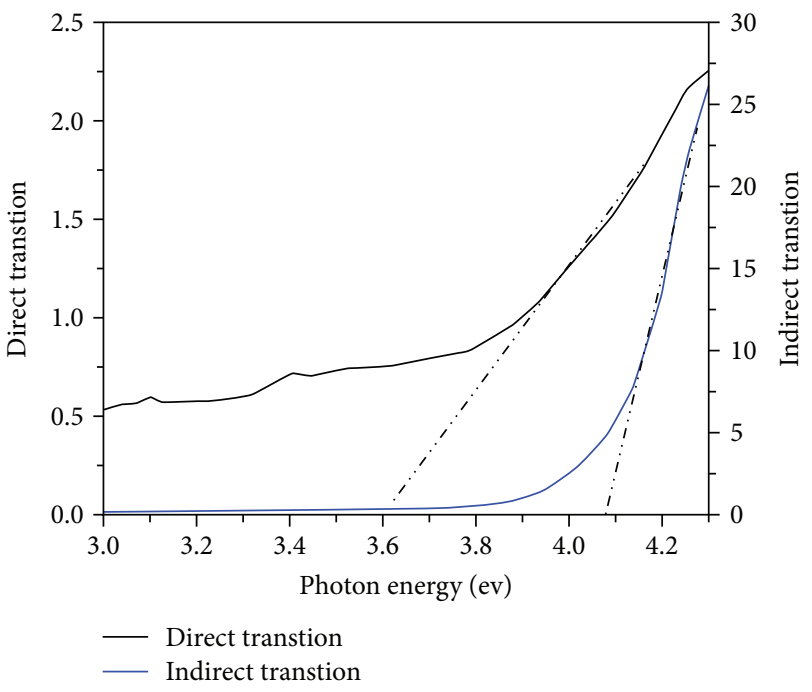

(b)

FIGURE 5: $(\alpha \mathrm{h} v)^{1 / 2}$ and $(\alpha \mathrm{h} v)^{2}$ versus photon energy of Si-NPs in (a) ethanol and (b) toluene.

different solvents is recorded at room temperature. The absorption coefficient, $E_{g}$ values, and reflectance of Si-NPs in different solvents are presented in Table 3. As observed in Table 3, the variation of absorption coefficient is depending on the nature of solvents and the solvent-solute interactions. The absorbance edge and band shapes of the spectrums are affected by the surrounding solvent environment. The absorbance increases with increasing the dielectric constant values of the reaction mixture. The reflectance values of Si-NPs in different solvents are varying because of different solvent properties, which affect the absorption edge and band shapes of the spectrum.

Figure 6 shows the correlation between polarity index and dielectric constant with emission peak. As shown in
Figure 6, the absorption coefficient increases because of high polarity index of solvents.

The extinction coefficient $(K)$ was obtained to measure the fraction of light lost due to scattering and absorption per unit distance of the penetration medium. The extinction coefficient is computed in the sample during the exposure of UV spectra by using the relation between \% absorption and wavelength [25]. Extinction coefficient $(K)$ is given by

$$
K=\alpha \frac{\lambda}{4} \pi
$$

where $\alpha$ is $\%$ absorption and $\lambda$ is wavelength. 
TABLE 3: List of absorption coefficient, $E_{g}$ values, and reflectance of Si-NPs in different solvents.

\begin{tabular}{|c|c|c|c|c|c|}
\hline Solvents & $\begin{array}{l}\text { Absorption coefficient } \alpha \\
\left(\mathrm{cm}^{-1}\right)\end{array}$ & $\begin{array}{l}\text { Peak maxima }(\lambda) \\
(\mathrm{nm})\end{array}$ & $\begin{array}{l}\mathrm{h} v \\
(\mathrm{eV})\end{array}$ & $\begin{array}{l}\mathrm{x} E_{g} \\
(\mathrm{eV})\end{array}$ & $\begin{array}{l}\text { Minimum reflectance narrow window } \\
\qquad(\mathrm{nm})\end{array}$ \\
\hline DMSO & 2.17 & 272 & 4.56 & 2.72 & $272-280$ \\
\hline DMF & 1.85 & 276 & 4.50 & 2.25 & $272-292$ \\
\hline Acetonitrile & 2.076 & 228 & 5.44 & 3.37 & $\begin{array}{l}224-228 \\
248-264\end{array}$ \\
\hline Ethanol & 1.968 & 220 & 5.64 & 3.52 & $276-288$ \\
\hline Dioxane & 1.804 & 284 & 4.37 & 3.26 & $\begin{array}{l}216-224 \\
256-264\end{array}$ \\
\hline Ethyl acetate & 0.668 & 256 & 4.85 & 2.52 & $252-256$ \\
\hline Chloroform & 1.821 & 248 & 5 & 2.95 & $\begin{array}{l}244-252 \\
336-360\end{array}$ \\
\hline THF & 1.892 & 280 & 4.43 & 3.93 & $272-288$ \\
\hline 1,2-Dichloroethane & 1.491 & 288 & 4.31 & 3.29 & $\begin{array}{l}284-292 \\
332-368\end{array}$ \\
\hline Benzene & 1.247 & 284 & 4.37 & 3.78 & $280-292$ \\
\hline Toluene & 1.21 & 288 & 4.31 & 3.61 & $284-292$ \\
\hline $\begin{array}{l}\text { Carbon } \\
\text { tetrachloride }\end{array}$ & 2.396 & 272 & 4.56 & 3.52 & $264-272$ \\
\hline Trichloroethylene & 0.898 & 280 & 4.43 & 3.49 & $\begin{array}{l}276-288 \\
356-386\end{array}$ \\
\hline Hexane & 2.056 & 244 & 5.1 & 3.56 & $228-244$ \\
\hline
\end{tabular}

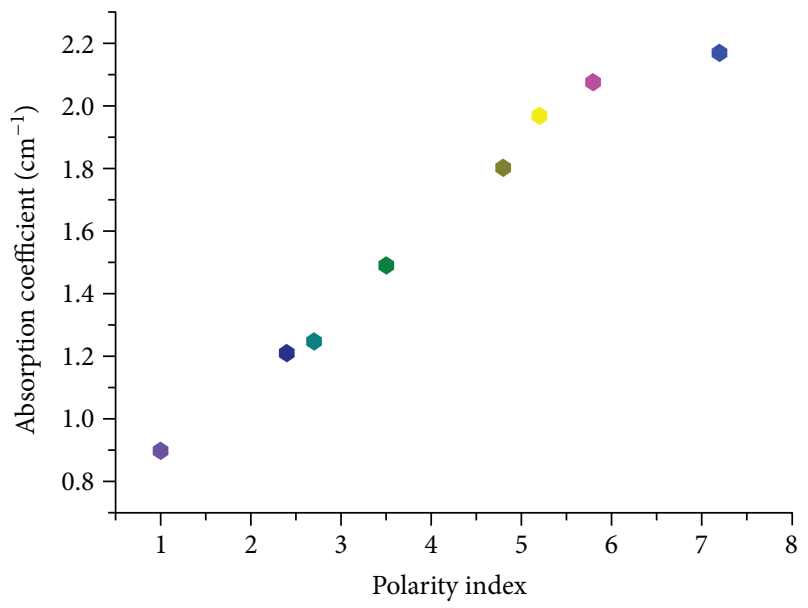

Figure 6: Variation of absorption coefficient for Si-NPs in different solvents with polarity index.

Figure 7 exhibits the extinction coefficient of Si-NPs in solvents which increases with photon energy and decreases with wavelength. The extinction coefficient describes the properties of the material with respect to light of a given wavelength and indicates the absorption changes when the electromagnetic wave propagates through the material. In Figure 7, the extinction coefficient of Si-NPs in solvents has a peak at different values as seen in Table 4,which increases with increasing concentration of Si-NPs in dielectric solvent medium.

\section{Conclusion}

Si-NPs were successfully prepared by chemical etching method and suspended in various solvents to investigate the impact of solvents (DMSO, DMF, ethanol, benzene, hexane, $\mathrm{CCl}_{4}$, chloroform, acetonitrile, etc.) on optical properties of Si-NPs. The absorption band edge and shape of band were varied causing the nature of chemical interaction between the Si-NPs and solvent. The maximum emission intensity of Si-NPs was observed in all solvents under monitoring excitation at $355 \mathrm{~nm}$. Two maximum intensity emission peaks were observed located at $400 \mathrm{~nm}$ and $600 \mathrm{~nm}$ on excitation at $355 \mathrm{~nm}$; the observed emission band shifting is due to the nature of solvent and etched cross-section area of the surface. Solvents have shown considerable effects on the emission wavelengths. Optical parameters such as absorption coefficient, absorption edge, transmittance, reflectance, extinction coefficient, and dielectric constant suggested the impact of solvent on the optical behavior of the SiNPs. The nature of solvent and their interaction with Si-NPs influenced the optical parameters and emission intensity. The increase in $E_{g}$ values is directly related to the decrease particle size, which is directly dependent on Si-NP size and the polarity of the solvent. The emission and absorption properties of Si-NPs were tuned by altering the environment (solvents) through electrostatic interaction of various organic solvents with the Si-NPs. The band shapes of the Si-NPs show remarkable changes in passing from noncoordinating solvent (chloroform) to various coordinating solvents, which is the result of change in the environment around Si-NPs in various solutions. 


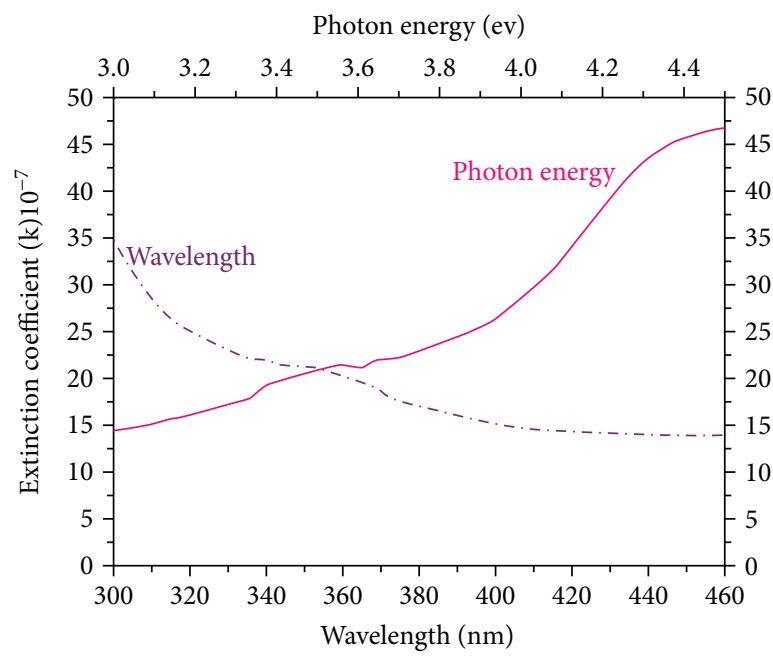

(a)

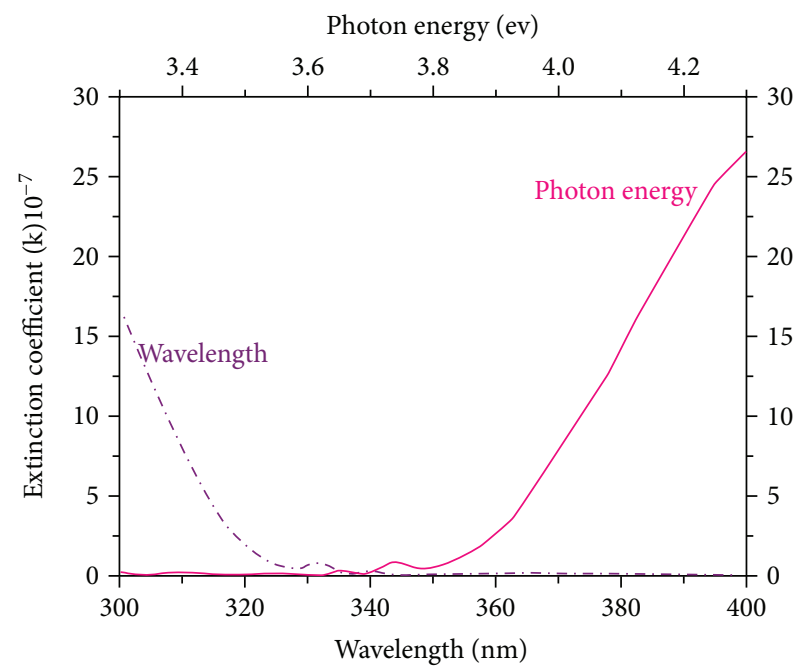

(c)

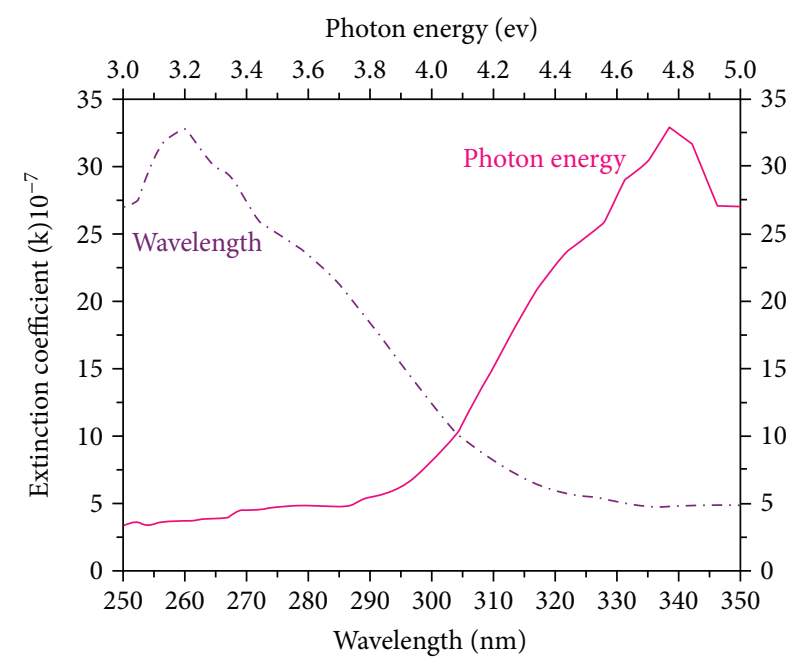

(b)

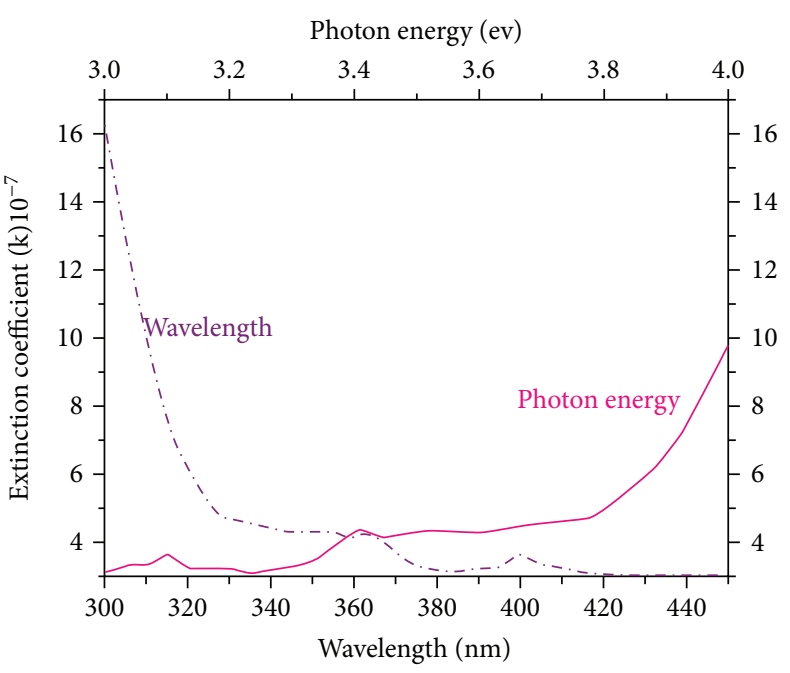

(d)

Figure 7: The photon energy dependence of extinction coefficient of Si-NPs in (a) DMSO, (b) ethanol, (c) benzene, and (d) toluene.

TABle 4: The maximum and minimum values of the extinction coefficient at the band gap region of Si-NPs in different solvents.

\begin{tabular}{|c|c|c|c|c|c|c|c|c|c|}
\hline Solvents & $\begin{array}{l}k_{\max } \\
10^{-7}\end{array}$ & $\lambda(\mathrm{nm})$ & $\mathrm{h} v(\mathrm{eV})$ & $\begin{array}{l}k_{\min } \\
10^{-7}\end{array}$ & $\lambda(\mathrm{nm})$ & $\mathrm{h} v(\mathrm{eV})$ & $\begin{array}{c}k \\
10^{-7}\end{array}$ & $\lambda(\mathrm{nm})$ & $\mathrm{h} v(\mathrm{eV})$ \\
\hline DMSO & 46.97 & 272 & 4.56 & 8.75 & 216 & 5.74 & 13.90 & 456 & 2.72 \\
\hline DMF & 49.20 & 600 & 2.10 & 15.9 & 192 & 6.46 & 45.95 & 552 & 2.25 \\
\hline Acetonitrile & 37.70 & 228 & 5.44 & 0.68 & 500 & 2.48 & 1.64 & 368 & 3.37 \\
\hline Ethanol & 34.45 & 220 & 5.64 & 3.41 & 416 & 2.98 & 4.82 & 352 & 3.52 \\
\hline Dioxane & 40.80 & 284 & 4.37 & 3.03 & 216 & 5.74 & 7.83 & 380 & 3.26 \\
\hline Ethyl acetate & 13.61 & 256 & 4.85 & 3.41 & 204 & 6.10 & 14.4 & 492 & 2.52 \\
\hline Chloroform & 35.90 & 248 & 5.00 & 0.33 & 584 & 2.21 & 1.57 & 420 & 2.95 \\
\hline THF & 42.16 & 280 & 4.43 & 2.31 & 236 & 5.26 & 22.45 & 316 & 3.93 \\
\hline 1,2-Dichloroethane & 34.17 & 288 & 4.31 & 0.91 & 188 & 6.60 & 9.69 & 376 & 3.29 \\
\hline Benzene & 28.18 & 284 & 4.37 & 0.00 & 388 & 3.20 & 0.42 & 328 & 3.78 \\
\hline Toluene & 27.73 & 288 & 4.31 & 1.23 & 228 & 5.44 & 4.30 & 344 & 3.61 \\
\hline Carbon tetrachloride & 51.86 & 272 & 4.56 & 7.98 & 244 & 5.08 & 29.44 & 352 & 3.52 \\
\hline Trichloroethylene & 20.16 & 284 & 4.37 & 0.00 & 600 & 2.07 & 3.37 & 356 & 3.49 \\
\hline Hexane & 39.92 & 244 & 5.08 & 0.22 & 340 & 3.65 & 1.22 & 348 & 3.56 \\
\hline
\end{tabular}




\section{Conflicts of Interest}

The authors declare that they have no conflicts of interest.

\section{Acknowledgments}

The authors would like to thank the Deanship of Scientific Research, College of Science King Saud University for funding this work.

\section{References}

[1] G. Belomoin, J. Therrien, and M. Nayfeh, "Oxide and hydrogen capped ultrasmall blue luminescent Si nanoparticles," Applied Physics Letters, vol. 77, no. 6, pp. 779-781, 2000.

[2] G. Belomoin, J. Therrien, A. Smith et al., "Observation of a magic discrete family of ultrabright Si nanoparticles," Applied Physics Letters, vol. 80, no. 5, pp. 841-843, 2002.

[3] B. Kim, D. Kim, C. Lee, and N. Min, "Photoluminescence from Nano silicon materials prepared by photoelectrochemical methods," Journal of the Korean Physical Society, vol. 38, no. 3, pp. 245-250, 2001.

[4] A. Uhlir Jr., "Electrolytic shaping of germanium and silicon," The Bell System Technical Journal, vol. 35, no. 2, pp. 333-347, 1956.

[5] D. R. Turner, "Electropolishing silicon in hydrofluoric acid solutions," Journal of the Electrochemical Society, vol. 105, no. 7, p. 402, 1958.

[6] L. T. Canham, "Silicon quantum wire array fabrication by electrochemical and chemical dissolution of wafers," Applied Physics Letters, vol. 57, no. 10, pp. 1046-1048, 1990.

[7] W. C. Chan and S. Nie, "Quantum dot bioconjugates for ultrasensitive nonisotopic detection," Science, vol. 281, no. 5385, pp. 2016-2018, 1998.

[8] M. Bruchez Jr., M. Moronne, P. Gin, S. Weiss, and A. P. Alivisatos, "Semiconductor nanocrystals as fluorescent biological labels," Science, vol. 281, no. 5385, pp. 2013-2016, 1998.

[9] T. D. Lacoste, X. Michalet, F. Pinaud, D. S. Chemla, A. P. Alivisatos, and S. Weiss, "Ultrahigh-resolution multicolor colocalization of single fluorescent probes," Proceedings of the National Academy of Sciences of the United States of America, vol. 97, no. 17, pp. 9461-9466, 2000.

[10] X. Michalet, F. F. Pinaud, L. A. Bentolila et al., "Quantum dots for live cells, in vivo imaging, and diagnostics," Science, vol. 307, no. 5709, pp. 538-544, 2005.

[11] J. K. Jaiswal and S. M. Simon, "Potentials and pitfalls of fluorescent quantum dots for biological imaging," Trends in Cell Biology, vol. 14, no. 9, pp. 497-504, 2004.

[12] M. Naziruddin Khan and A. S. Al Dwayyan, "Influence of solvent on the physical and lasing properties of dye-doped sol-gel host," Journal of Luminescence, vol. 128, no. 11, pp. 1767-1770, 2008.

[13] N. Wang, Y. H. Tang, Y. F. Zhang, C. S. Lee, and S. T. Lee, "Nucleation and growth of Si nanowires from silicon oxide," Physical Review B, vol. 58, no. 24, pp. R16024-R16026, 1998.

[14] S. T. Lee, N. Wang, and C. S. Lee, "Semiconductor nanowires: synthesis, structure and properties," Materials Science and Engineering: A, vol. 286, no. 1, pp. 16-23, 2000.
[15] S. T. Lee, N. Wang, Y. F. Zhang, and Y. H. Tang, "Oxideassisted semiconductor nanowire growth," MRS Bulletin, vol. 24, no. 8, pp. 36-42, 1999.

[16] N. O’Farrell, A. Houlton, and B. R. Horrocks, "Silicon nanoparticles: applications in cell biology and medicine," International Journal of Nanomedicine, vol. 1, no. 4, pp. 451-472, 2006.

[17] B. O. Dabbousi, M. G. Bawendi, O. Onitsuka, and M. F. Rubner, "Electroluminescence from CdSe quantum-dot/ polymer composites," Applied Physics Letters, vol. 66, no. 11, pp. 1316-1318, 1995.

[18] M. Naziruddin Khan, M. A. Majeed Khan, A. S. Al Dwayyan, and J. Puzon Labis, "Comparative study on electronic, emission, spontaneous property of porous silicon in different solvents," Journal of Nanomaterials, vol. 2014, Article ID 682571, 14 pages, 2014.

[19] A. Aldwayyan, A. Ali, A. A. Ansari, M. S. Alsalhi, and M. H. Nayfeh, "Effect of environments on optical properties of chemically prepared Si nanoparticles," Advanced Science, Engineering and Medicine, vol. 5, no. 9, pp. 965-970, 2013.

[20] M. H. Nayfeh, E. V. Rogozhina, and L. Mitas, Synthesis Functionalization and Surface Treatment of Nanoparticles, American Scientific Publishers, Stevenson Ranch, CA, USA, 2003, Chapter 10.

[21] V. Gutmann, "Solvent effects on the reactivities of organometallic compounds," Coordination Chemistry Reviews, vol. 18, no. 2, pp. 225-255, 1976.

[22] A. A. Ansari, N. Singh, A. F. Khan, S. P. Singh, and K. Iftikhar, "Solvent effect on optical properties of hydrated lanthanide tris-acetylacetone," Journal of Luminescence, vol. 127, no. 2, pp. 446-452, 2007.

[23] S. Fellah, R. B. Wehrspohn, N. Gabouze, F. Ozanam, and J. N. Chazalviel, "Photoluminescence quenching of porous silicon in organic solvents: evidence for dielectric effects," Journal of Luminescence, vol. 80, no. 1-4, pp. 109-113, 1998.

[24] T. Tauc and F. Abeies, Optical Properties of Solids, IOP Publishing Ltd, North Holland, Amsterdam, 1970.

[25] N. A. Bakr, A. M. Funde, V. S. Waman et al., "Determination of the optical parameters of a-Si:H thin films deposited by hot wire-chemical vapour deposition technique using transmission Spectrum only," Pramana, vol. 76, no. 3, pp. 519-531, 2011. 

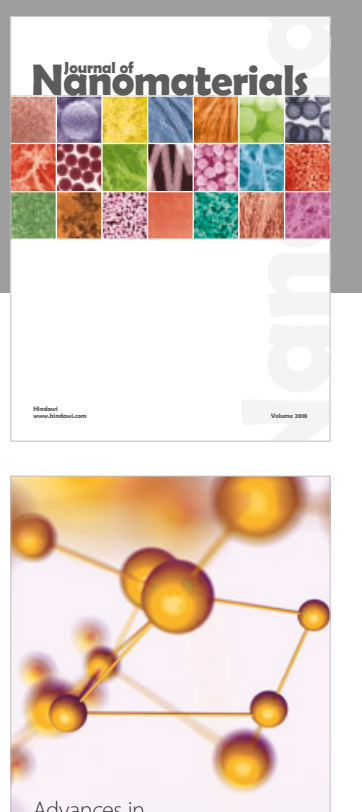

Physical Chemistry
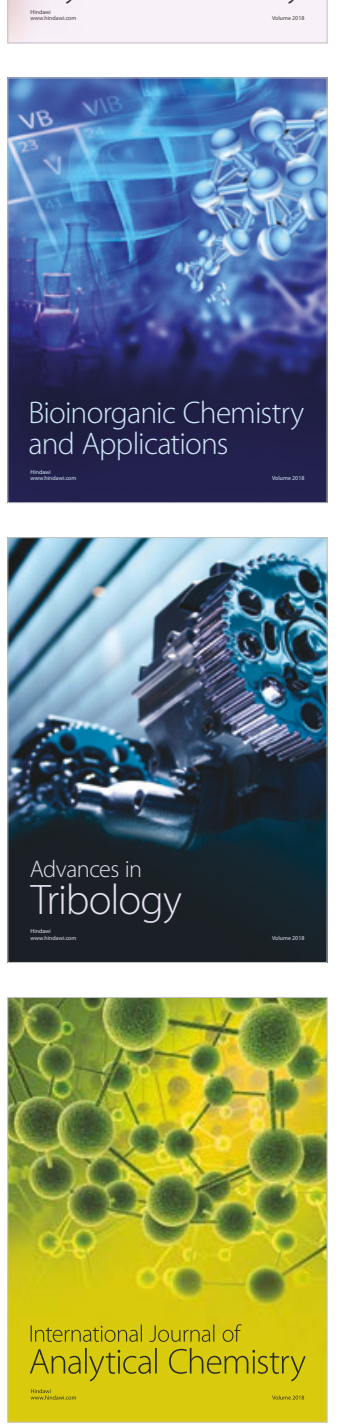

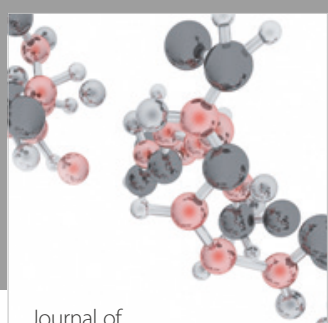

Analytical Methods

in Chemistry

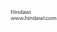

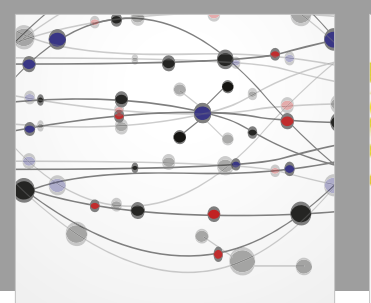

The Scientific World Journal

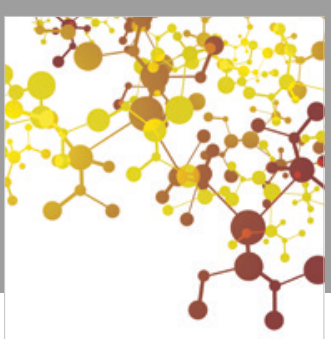

Journal of

Applied Chemistry
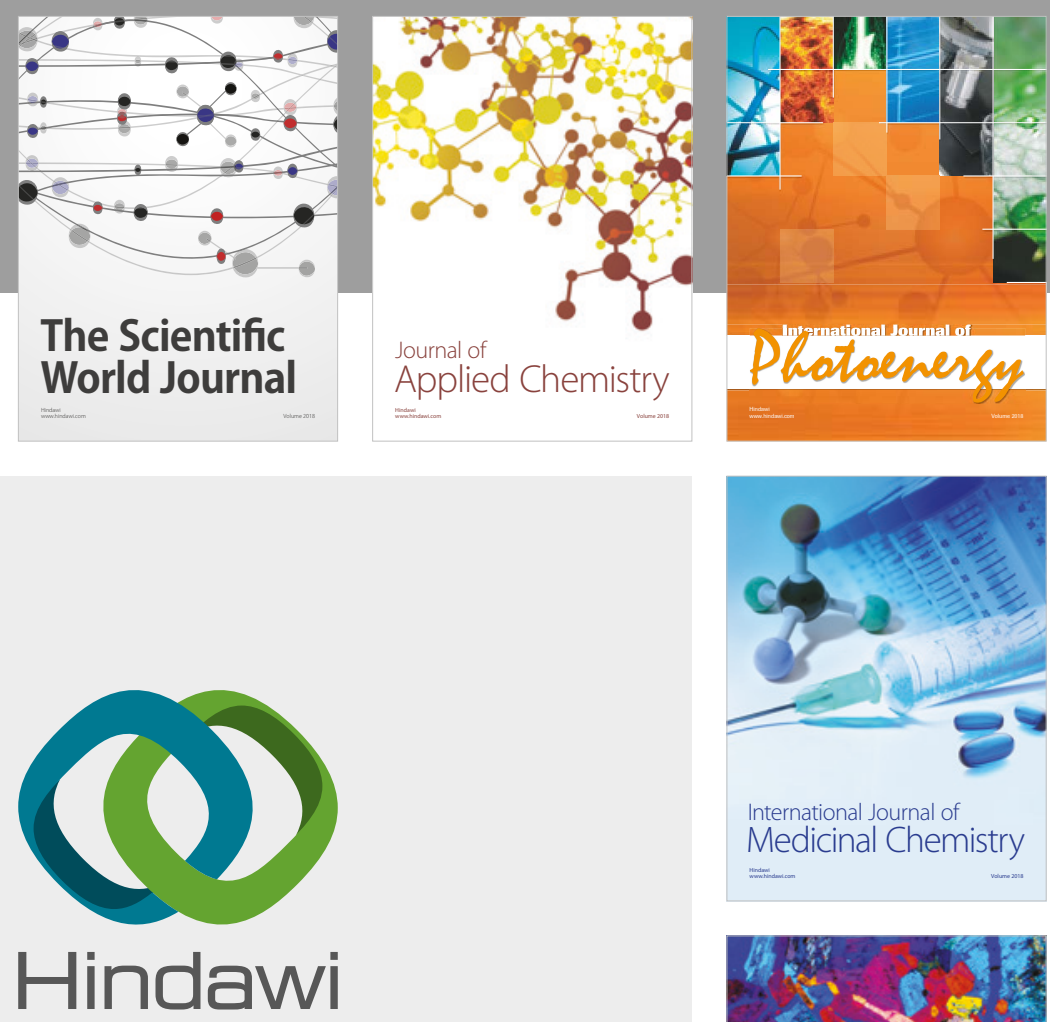

Submit your manuscripts at

www.hindawi.com
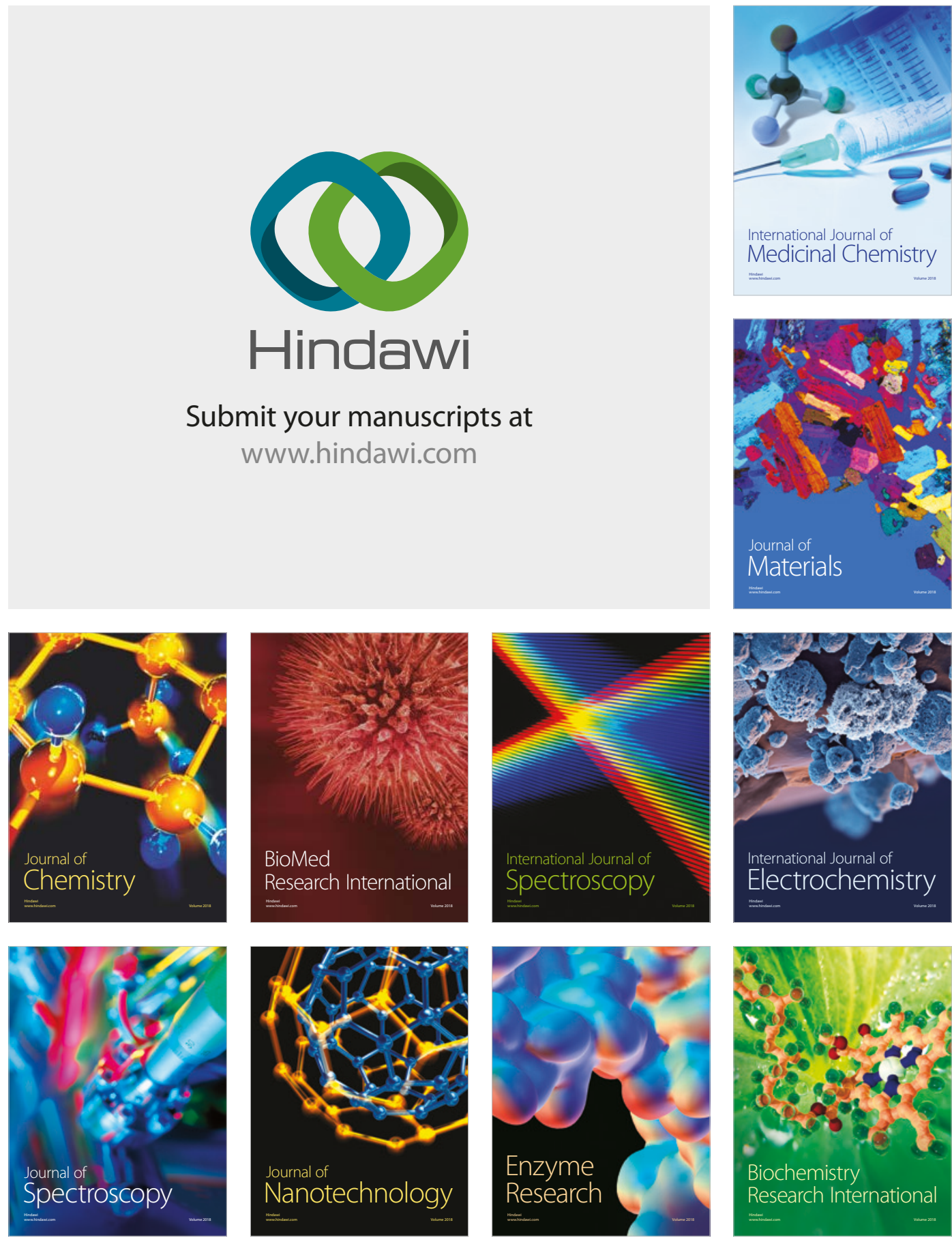
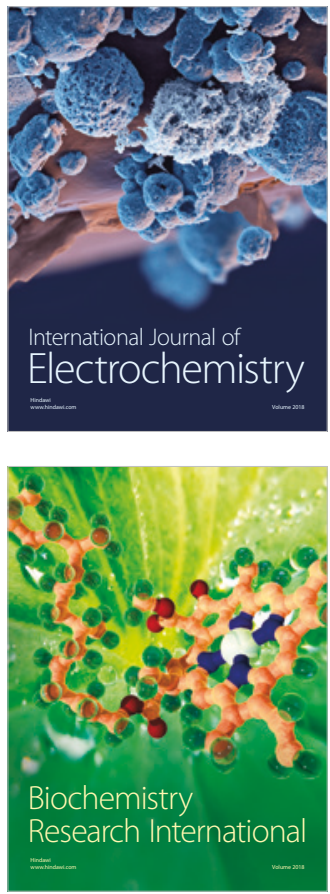